\title{
Politique
}

Politique

\section{Être contemporain. Mélanges en l'honneur de Gérard Bergeron sous la direction de Jean-William Lapierre, Vincent Lemieux et Jacques Zylberberg, Sillery, Presses de l’Université du Québec, 1992, 518 p.}

\section{Micheline de Sève}

Numéro 23, hiver 1993

Tendances de la science politique au Québec

URI : https://id.erudit.org/iderudit/040756ar

DOI : https://doi.org/10.7202/040756ar

Aller au sommaire du numéro

Éditeur(s)

Société québécoise de science politique

ISSN

0711-608X (imprimé)

1918-6584 (numérique)

Découvrir la revue

Citer ce compte rendu

de Sève, M. (1993). Compte rendu de [Être contemporain. Mélanges en l'honneur de Gérard Bergeron sous la direction de Jean-William Lapierre, Vincent Lemieux et Jacques Zylberberg, Sillery, Presses de l’Université du Québec, 1992, 518 p.] Politique, (23), 226-228. https://doi.org/10.7202/040756ar d'utilisation que vous pouvez consulter en ligne. 


\section{Etre contemporain. Mélanges en l'honneur de Gérard Bergeron}

sous la direction de Jean-William Lapierre, Vincent Lemieux et Jacques Zylberberg, Sillery, Presses de l'Université du Québec, 1992, 518 p.

Après l'hommage à Léon Dion, voici un second ouvrage consacré à l'un des grands de la science politique québécoise, le maître du paradoxe : Gérard Bergeron. L'entreprise étant hasardeuse qui veuille retenir toutes les facettes de la production du théoricien, de I'historien ou du journaliste, ces personnages que Bergeron excelle à jouer avec une virtuosité proprement inimitable. Encore a-t-on ignoré le chantre de la chanson québécoise, Alain Sylvain, à qui Bergeron prêta sa voix pour une série d'émissions radiophoniques passionnantes au cours des années soixante. La fantaisie laisse ici place à un ouvrage sagement cloisonné en quatre parties distinctes. La première est consacrée à la critique des théories de celui dont Raymond Aron saluait la légitime ambition au moment de préfacer Fonctionnement de l'État en 1965 et qui ne cessera de stimuler la recherche autour de lui dans des directions aussi multiples que les intérêts des collègues attachés à développer l'une ou l'autre de ses propositions. Ce n'est pas un mince crédit rendu à l'œuvre de Bergeron que cette capacité d'inspirer aussi bien des spécialistes de l'analyse politique comme Jean-William Lapierre, Daniel-Louis Seiler, 
James D. Driscoll et Jacques Gagnon; des experts en communications, comme Jacques Rivet; en administration publique, comme Lionel Ouellet ou James lain Gow; en relations internationales, comme André Donneur et Daniel Colard; ou des méthodologues comme Réjean Landry.

La seconde partie, plus inégale, rassemble des textes où pour avoir collé de trop près à l'actualité, des auteurs présument à tort de la ratification de l'Accord du lac Meech, et d'autres chapitres, tout à fait remarquables,dont ceux rédigés par J. A. Laponce et Jean Tournon, qui traitent de l'ethnicité avec une finesse et un doigté rarement égalés.

La troisième partie, sur l'environnement contemporain, est forcément disparate. Nous mentionnerons en premier lieu d'excellentes études philosophiques comme celle de Louise Marcil-Lacoste lla seule femme à figurer au nombre des collaborateurs de l'ouvrage) sur la compatibilité de la liberté et de l'égalité en regard d'une critique de la possessivité chez Paine, ou de Léo Moulin, sur la fin des utopies. Mais d'autres chapitres sont constitués de commentaires moins heureux de l'actualité comme celui qui porte sur «L'évolution des Balkans et de I'URSS et où Jean-Louis Seurin développe le point de vue serbe sur le Kosovo, kenvahi par une immigration albanaise incontrôlable» (p. 417) pour ensuite avancer avec une belle certitude que $\alpha l^{\prime}$ actualité journalistique tend à exagérer la portée de "I'éclatement de l'URSS" " (p. 420). N'est pas sphynx qui veut et les émules de Bergeron ne partagent pas tous l'acuité de sa vision!

Reste que la plupart des chapitres des sections scientifiques de cet ouvrage composite se révéleront utiles à une clientèle politologique avisée. Et curieuse de découvrir des pistes de recherches inattendues comme celle ouverte par Réjean Landry qui n'a pas hésité à formaliser et simuler par ordinateur la théorie exposée par Gérard Bergeron dans La gouverne politique ou Jacques Zylberberg qui provoque la réflexion avec ses «Notes sur la gestion fractale des liens sociaux dans la post-modernitéw. Enfin, la dernière partie de l'ouvrage nous rapproche de l'homme à travers les aspects multiples d'une personnalité aussi riche que secrète, à peine devinée par ses anciens étudiants ou collègues. Le tout est déroutant par moments mais séduira les adeptes d'une pensée 
ouverte et versatile comme celle de Gérard Bergeron luimême.

Micheline de Sève

Université du Québec à Montréal 